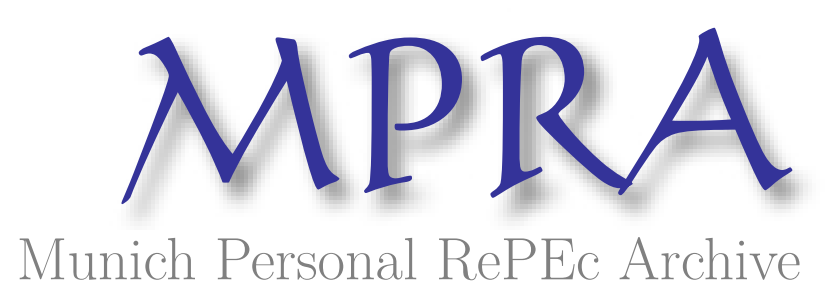

Shape Factor Asymptotic Analysis I

Wang, Frank Xuyan

2019

Online at https://mpra.ub.uni-muenchen.de/93357/

MPRA Paper No. 93357, posted 24 Apr 2019 02:56 UTC 


\title{
Shape Factor Asymptotic Analysis I
}

\author{
Xuyan Frank Wang [0000-0001-7653-5755] \\ Validus Research Inc., 187 King Street South Unit 201, Waterloo, Ontario, Canada N2J 1R1 \\ frank.wang@validusresearch.com
}

\begin{abstract}
The shape factor defined as kurtosis divided by skewness squared $\frac{K}{S^{2}}$ is characterized as the only choice among all factors $\frac{K}{|S|^{\alpha}}, \alpha>0$ which is greater than or equal to 1 for all probability distributions. For a specific distribution family, there may exists $\alpha>2$ such that $\min \frac{K}{|S|^{\alpha}} \geq 1$. The least upper bound of all such $\alpha$ is defined as the distribution's characteristic number. The useful extreme values of the shape factor for various distributions which are found numerically before, the Beta, Kumaraswamy, Weibull, and GB2 Distribution, are derived using asymptotic analysis. The match of the numerical and the analytical results can be considered prove of each other. The characteristic numbers of these distributions are also calculated. The study of the boundary value of the shape factor, or the shape factor asymptotic analysis, help reveal properties of the original shape factor, and reveal relationship between distributions, such as between the Kumaraswamy distribution and the Weibull distribution.
\end{abstract}

Keywords: Shape Factor, Skewness, Kurtosis, Asymptotic Expansion, Beta Distribution, Kumaraswamy Distribution, Weibull Distribution, GB2 Distribution, Computer Algebra System, Numerical Optimization, Characteristic Number.

\section{Introduction}

The concept of shape factor is proposed and studied for various probability distribution families [1][2] ([1] has more background and references, [2] is more cogent). Three kind of uses are made of the shape factor: the global lower bound of the shape factor for a distribution family can be used to eliminate those distributions for data fitting that have these bound higher than the data distribution; when these bounds are not violated, the plot of the minimum shape factor value for given parameter can be used to locate the allowable range of that parameter; combine the shape factor plot with skewness plot, for known sign of the skewness, the allowable parameters ranges can be identified. Since in practice we mostly see positive skewness, we will generally restrict our analysis to the positive skewness region.

Numerical optimizations are used to get plots of those minimum shape factors. There are two types of possibly errors in those numerical studies: the system error and the operational error. The first type error is bringing about by the software system that performing those analysis, such as the algorithms shortcomings used for the transcendental 
functions, or the allowable numerical range for machine-precision numbers and arbitrary-precision numbers. The remedy for the system error is to check by software that are using different under-the-hood implementations. The operational or human error is occurred when manipulating the mathematical expressions, such as using not exactly equivalent substitutions or transformations. To reduce this kind of error, multiple approaches need to undertake to validate each other. The numerical and graphical results need to be subsidized by analytical deductions.

When the minimum of the shape factor is attained at the region interior, it can be found from the zero point of the partial derivatives. This is an application of differential analysis and root finding. The other case is at the boundary, usually either at 0 or infinity, an application of limit and asymptotic expansion/analysis.

We will redo the mathematical analysis of the Beta, Kumaraswamy, GB2, GB1, and GH distributions shape factors, with either new formulas found, or more analytical ways to support our original empirical plots, adding more rigorousness to our conclusions. To avoid repetition, we will resort to [1] or [2] heavily for most of the omitted contents. The GB1, GH studies will be in a second paper due to page length limitation.

\section{Results}

\subsection{Shape Factor Characterization}

The shape factor is found and defined in Wang [1][2], where simple power and exponential forms of distributions examples are used to justify that definition. Here we find more reason for the uniqueness of this definition.

For a random variable $f$ with mean $m_{f}$, the following characteristics are defined:

- Moment (M), $M[r] \equiv \int f^{r} d \mu, r>0$,

- Central Moment (CM), $C M[r] \equiv \int\left(f-m_{f}\right)^{r} d \mu, r>0$,

- Absolute Central Moment (ACM), ACM $[r] \equiv \int\left|f-m_{f}\right|^{r} d \mu, r>0$,

- Skewness (S), $S \equiv \frac{C M[3]}{C M[2]^{3}}$,

- Kurtosis $(\mathrm{K}), K \equiv \frac{C M[4]}{C M[2]^{2}}$

- Shape Factor (SF), $S F \equiv \frac{K}{S^{2}}=\frac{C M[4] * C M[2]}{C M[3]^{2}}$,

- $S F 3[r] \equiv \frac{A C M[r]}{A C M[1]^{r}} \leq 1$, where $0<r<1$ and $S F 3[s] \equiv \frac{A C M[s]}{A C M[1]^{s}} \geq 1$, where $s>1$,

- Standard Deviation (SD), $S D \equiv A C M[2]^{\frac{1}{2}}$,

- Absolute Mean Deviation (MD), $M D \equiv A C M[1]$,

- $S F 3[2]=\left(\frac{S D}{M D}\right)^{2}$.

By Jensen's inequality (https://en.wikipedia.org/wiki/Jensen's_inequality) we have:

$$
\left(\int\left(f-m_{f}\right)^{2} d \mu\right)^{2} \leq \int\left(f-m_{f}\right)^{4} d \mu, K \geq 1 .
$$


From [1], we also know that $K \geq S^{2}, K \geq S^{2} \geq|S|$ if $|S| \geq 1, K \geq 1>|S|$ if $|S|<$ 1. So we arrive at the following:

$$
K \geq|S|, \frac{K}{|S|} \geq 1 .
$$

We can similarly get (by monotonicity of $|S|^{\alpha}$ w.r.t. $\alpha$ ):

$$
\begin{aligned}
& K \geq S^{\frac{4}{3}}, \frac{K}{S^{\frac{4}{3}}} \geq 1, \\
& K \geq|S|^{\alpha}, \frac{K}{|S|^{\alpha}} \geq 1, \text { if } 0 \leq \alpha \leq 2 .
\end{aligned}
$$

The equation (2)-(4) can be used where the quotient can give simpler forms. For example, if the central moment have simpler forms than the skewness and kurtosis, then (3) will be simpler, involving only $\frac{C M[4]}{C M[3]^{\frac{4}{3}}}$.

Equation (4) says that the shape factor is among an extended family of shape factors $\frac{K}{|S|^{\alpha}}$ that are bound below by 1 , so we will call all of them the shape factors.

We postulate that 2 is the least upper bound of all $\alpha$ such that $\frac{K}{|S|^{\alpha}} \geq 1$ hold for all distribution families (that is, the condition in equation (4) is not only sufficient, but also necessary). But for a specific distribution family this inequality may hold for $\alpha$ larger than 2. These statements will be proved by example cases in section 2.3.

We guess for each specific distribution family there exists a critical value of $\alpha$ which is not less than 2, such that above it the minimum of $\frac{K}{|S|^{\alpha}}$ will be 0 , and below it, the minimum of $\frac{K}{|S|^{\alpha}}$ will be bigger than 1 . We will call such a critical value where the minimums of the shape factors have a sharp jump the critical value or the characteristic number of the distribution.

The limit of the extended shape factors at 0 or infinity for parameters usually has simpler form that can be considered as a prototype, asymptotic value, or magnitude of order [3], in some cases are also the lower or upper bound, of the shape factors. The properties of these simpler form will give hint of similar properties for the original shape factors, such as for the critical value we guessed. We will start that limit calculation with the simplest distribution in the next section.

\subsection{Beta Distribution}

With the naming and parameterization convention for probability distributions from Mathematica or [4], for the BetaDistribution $[\alpha, \beta]$, we have

$S F=\frac{3(2+\alpha+\beta)\left(\alpha(-2+\beta) \beta+2 \beta^{2}+\alpha^{2}(2+\beta)\right)}{4(\alpha-\beta)^{2}(3+\alpha+\beta)}$.

For practical use, we only consider the parameters region where $S>0$, so in this case $\beta>\alpha$. 
For a fixed $\beta$, the lower boundary value at $\alpha=0$ is the minimum value of the shape factor:

$\operatorname{limit}_{\alpha \rightarrow 0} S F=\min _{0<\alpha<\beta} S F=\frac{3(2+\beta)}{2(3+\beta)}$.

This value increases from 1 to 1.5 when $\beta$ turns from 0 to $\infty$.

For a fixed $\alpha$, the upper boundary at $\beta=\infty$ and the minimum value of the shape factor are different:

$$
\begin{aligned}
& \min _{\beta>\alpha} S F=\frac{3 \alpha\left(4+4 \alpha-\alpha^{2}+\sqrt{\alpha\left(24+40 \alpha+16 \alpha^{2}+\alpha^{3}\right)}\right)}{4\left(6+\alpha-\alpha^{2}+\sqrt{\alpha\left(24+40 \alpha+16 \alpha^{2}+\alpha^{3}\right)}\right)\left(6 \alpha+5 \alpha^{2}+\sqrt{\alpha\left(24+40 \alpha+16 \alpha^{2}+\alpha^{3}\right)}\right)^{2}}(48+ \\
& 68 \alpha^{3}-\alpha^{4}+28 \sqrt{\alpha\left(24+40 \alpha+16 \alpha^{2}+\alpha^{3}\right)}+\alpha^{2}(232+ \\
& \left.\left.\sqrt{\alpha\left(24+40 \alpha+16 \alpha^{2}+\alpha^{3}\right)}\right)+16 \alpha\left(13+2 \sqrt{\alpha\left(24+40 \alpha+16 \alpha^{2}+\alpha^{3}\right)}\right)\right), \\
& \operatorname{limit}_{\beta \rightarrow \infty} S F=\frac{3(2+\alpha)}{4} .
\end{aligned}
$$

The upper boundary value of the shape factor for $\beta=\infty$ increases from 1.5 to $\infty$ when $\alpha$ turns from 0 to $\infty$. For given $\alpha$, the minimum value of the shape factor increases from 1 to 3 when $\alpha$ turns from 0 to $\infty$, Fig. 1 .

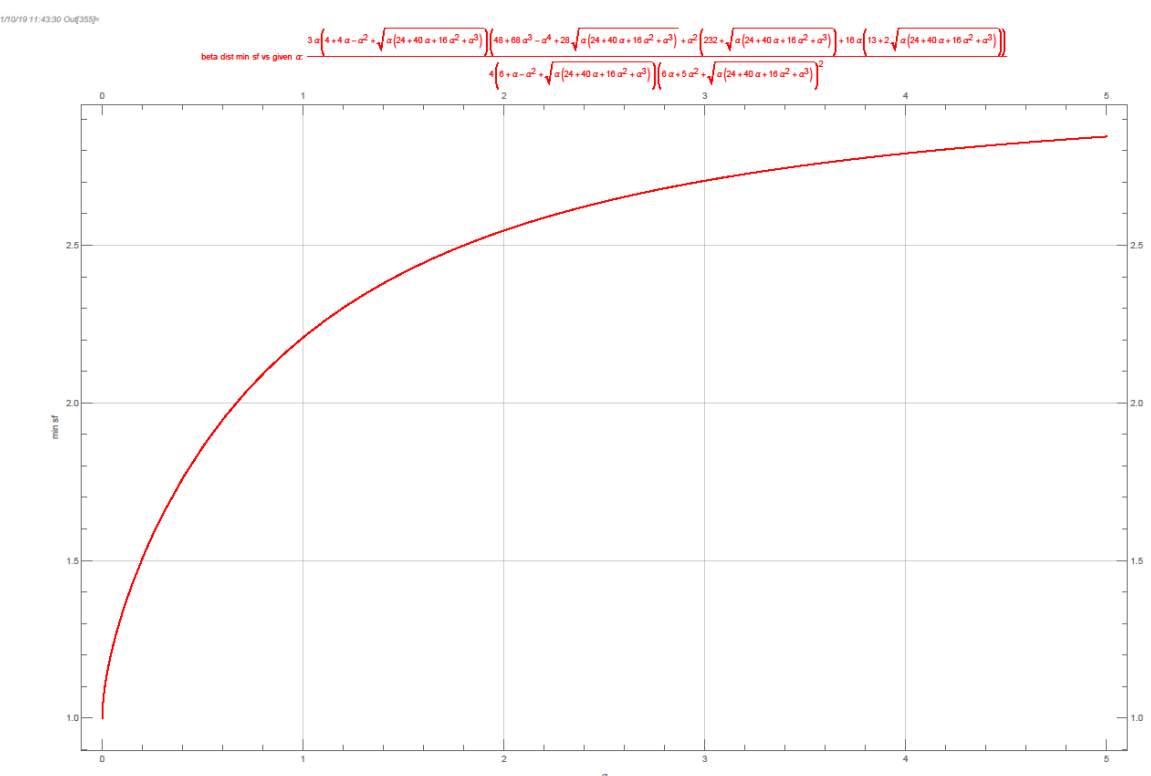

Fig. 1. Beta distribution minimum shape factor for given $\alpha$ in the horizontal axis.

For a fixed $\alpha$, the upper boundary value at $\beta=\infty$ and the maximum value of the skewness and the kurtosis are the same: 
$\operatorname{limit}_{\beta \rightarrow \infty} S=\max _{\beta>\alpha} S=\frac{2}{\sqrt{\alpha}}$

$\operatorname{limit}_{\beta \rightarrow \infty} K=\max _{\beta>\alpha} K=3+\frac{6}{\alpha}$

In practice, the equation (6) and (9) give relatively good (less than $10 \%$ error) upper bound estimate for the parameters $\beta$ and $\alpha$ from data $S F$ and $S$. This can be roughly stated as the skewness determines $\alpha$, the higher the skewness the smaller the $\alpha$, and the shape factor determines $\beta$, the higher the shape factor, the bigger the $\beta$. So in the Beta distribution case, the asymptotic analysis heuristically reveals the intrinsic meaning of the parameters: $\alpha$ for skewness and $\beta$ for shape factor.

\subsection{Kumaraswamy Distribution Part One}

\section{Given Skewness}

Even though more complex than Beta distribution, we will see that the limit and minimum value of the shape factor of the KumaraswamyDistribution $[\alpha, \beta]$ show similar pattern as the Beta distribution.

For given skewness, the extreme value of the shape factor or the kurtosis is attended at the boundary; this pattern is simpler than given $\alpha$ or $\beta$, and can be checked by the contour plot of the ratio of the skewness contour tangent to the kurtosis contour tangent, Fig.2. That ratio is always larger than 1 indicates that the maximum shape factor is at the top boundary when $\beta \rightarrow \infty$, and the minimum shape factor is at the left boundary when $\alpha \rightarrow 0$. 


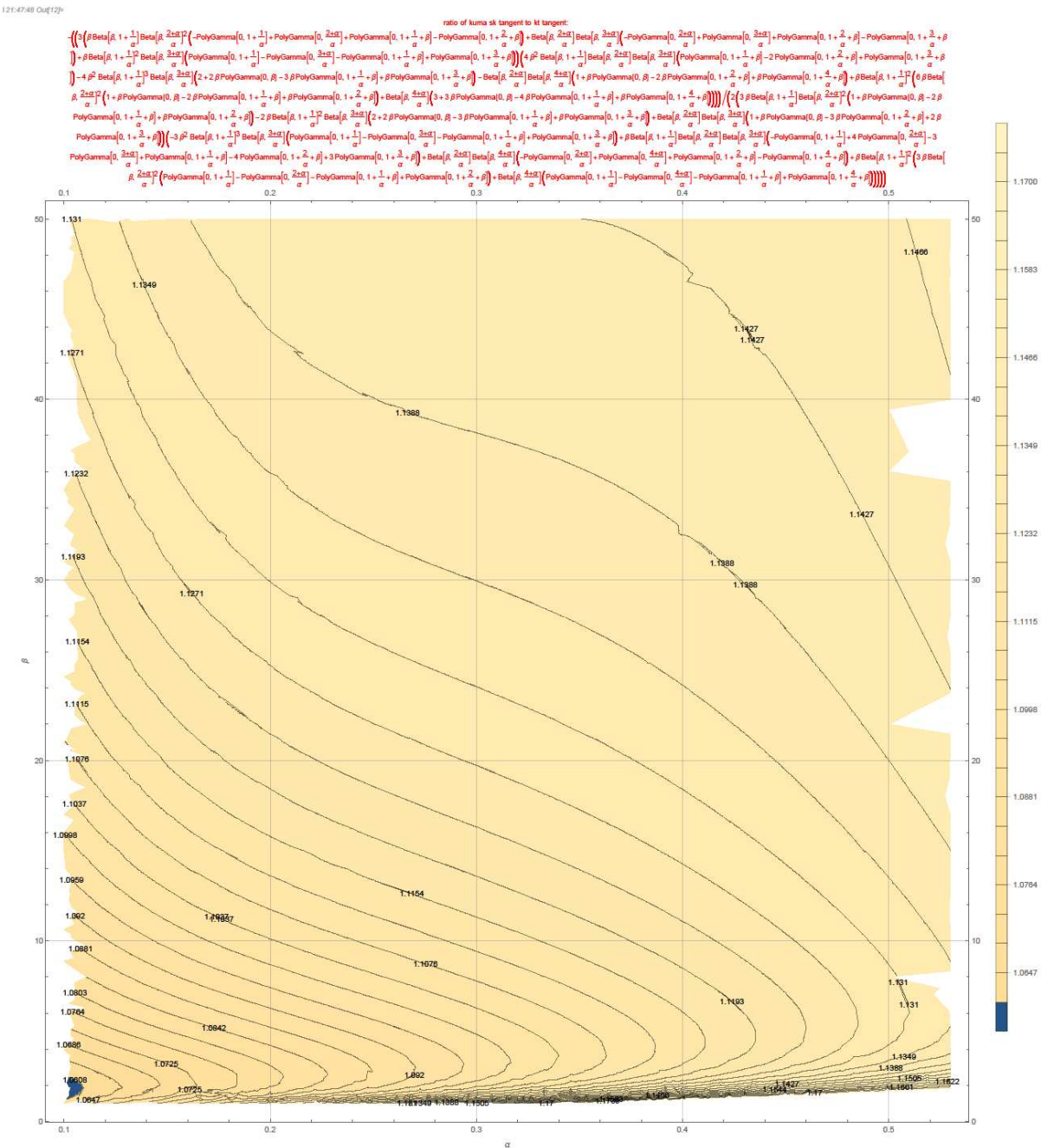

Fig. 2. Kumaraswamy distribution ratio of skewness contour tangent to kurtosis contour tangent plot.

This plot confirms that the limit when $\beta \rightarrow \infty$ gives the parametric representation of the maximum shape factor of Kumaraswamy distribution ([1] equation (4) and (6)). This maximum shape factor together with the broader distribution family GB1 [5] upper bound distribution $\operatorname{LogNormalDistribution}[\mu, \sigma]$ shape factor plot are in Fig. 3. 


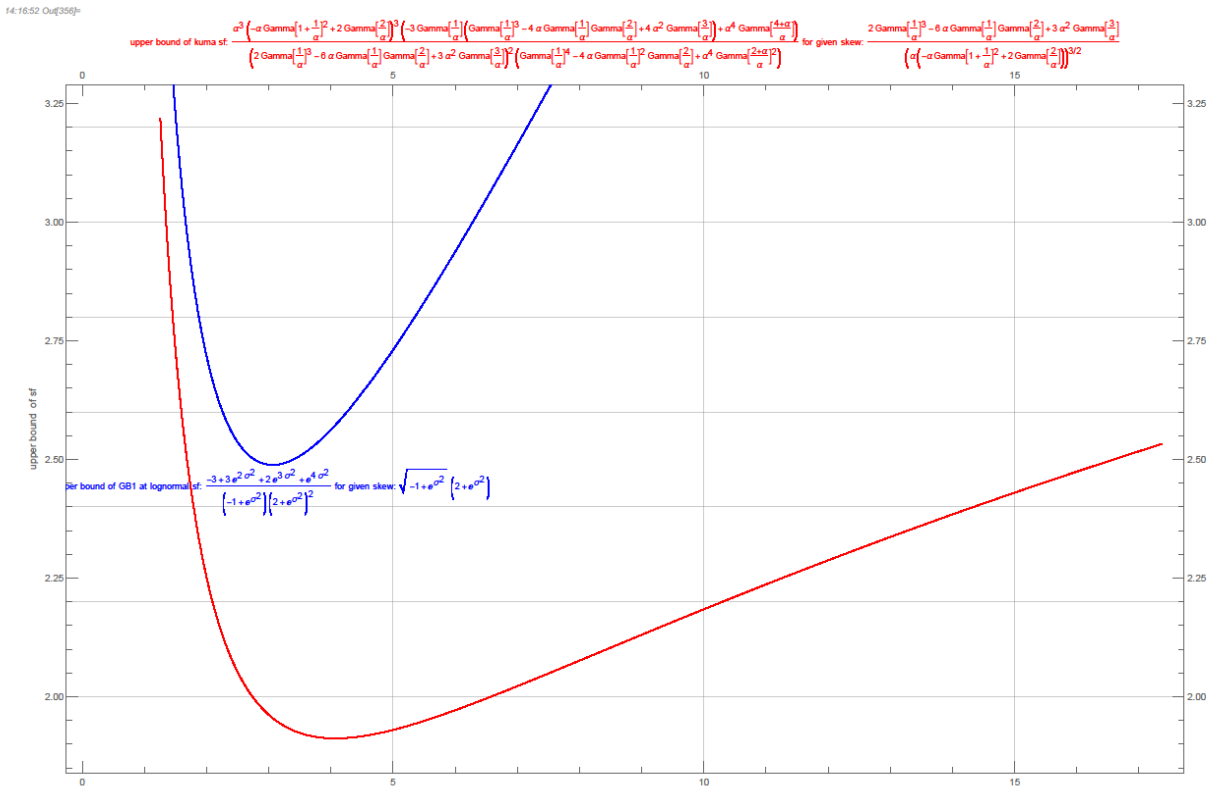

Fig. 3. Kumaraswamy distribution maximum shape factor and log normal distribution shape factor with respect to skewness plot.

\section{Boundary $\alpha=0$}

For given $\beta$, when $\alpha \rightarrow 0$, we will use the following $2^{\text {nd }}$ order asymptotic expansion at $\infty$ for $x$ :

$\operatorname{Beta}[\beta, x] \rightarrow \operatorname{Gamma}[\beta] \frac{1}{24} x^{-2-\beta}\left(24 x^{2}-12 x(-1+\beta) \beta+\beta\left(2-3 \beta-2 \beta^{2}+\right.\right.$ $\left.\left.3 \beta^{3}\right)\right)$.

To find the asymptotic order of a function, we will utilize the following heuristic trick:

if $\frac{d \log (f(x))}{d x} x \rightarrow \mathrm{a}$, then possibly $f(x) \sim x^{\mathrm{a}}$.

The computer algebra system (CAS) Mathematica may be able to find the first limit when it cannot prove the second formula. Combining these techniques, we finally get:

$\operatorname{limit}_{\alpha \rightarrow 0}\{S, K, S F\} \sim\left\{\frac{\left(\frac{2}{3}\right)^{\beta}}{\sqrt{2^{-\beta}\left(\frac{1}{\alpha}\right)^{-\beta} \beta \operatorname{Gamma}[\beta]}}, \frac{\left(\frac{1}{\alpha}\right)^{\beta}}{\beta \operatorname{Gamma}[\beta]},\left(\frac{9}{8}\right)^{\beta}\right\}$.

We see that skewness and kurtosis turn to infinity but the shape factor has finite limit. For $\mathrm{a}>0$, from equation (13), we have:

$$
\frac{\operatorname{limit}_{\alpha \rightarrow 0} K}{\operatorname{limit}_{\alpha \rightarrow 0} S^{\mathrm{a}}} \sim\left(\frac{1}{\alpha}\right)^{\left(1-\frac{\mathrm{a}}{2}\right) \beta} 2^{-\frac{3}{2} \mathrm{a} \beta} 3^{\mathrm{a} \beta}(\beta \operatorname{Gamma}[\beta])^{\frac{1}{2}(-2+\mathrm{a})} .
$$


From equation (14) we see that when a $>2, \operatorname{limit}_{\alpha \rightarrow 0} \frac{K}{S^{\mathrm{a}}} \sim 0$; when $0<\mathrm{a}<2, \operatorname{limit}_{\alpha \rightarrow 0} \frac{K}{S^{\mathrm{a}}} \sim \infty$; when a $=2, \operatorname{limit}_{\alpha \rightarrow 0} \frac{K}{S^{\mathrm{a}}} \sim\left(\frac{9}{8}\right)^{\beta}$. The Kumaraswamy distribution shows that the shape factor with $a=2$ is the only definition that gives nonzero and finite boundary shape factor values, and when a>2 this value is 0 . This conclusion is also true for the Beta distribution by equation (9) and (10).

This observation is not limited to the Kumaraswamy or the Beta distribution, for example, but valid also for the following kind of distribution with power function probability density function (PDF) $\frac{n+1}{n}\left(1-x^{n}\right), x \in[0,1], n>-1$, which is neither $\mathrm{Ku}$ maraswamy nor Beta distribution, it is also not GB1 as defined in [5], [6], or [4], having

$$
\begin{aligned}
S & =\frac{6 \sqrt{3}(1+n)(4+n(3+n))\left(\frac{3+n}{(1+n)(7+n(4+n))}\right)^{3 / 2}}{(3+n)(4+n)}, \\
K & =\frac{9(3+n)(572+n(1011+n(813+n(366+n(102+n(15+n))))))}{5(1+n)(4+n)(5+n)(7+n(4+n))^{2}},
\end{aligned}
$$

When $n \rightarrow-1$, only SF (used a=2) converges to a nonzero finite number 1.2. So these three types of distributions all have characteristic number 2 . These examples are proofs of our postulation in section 2.1 .

\subsection{Weibull Distribution}

If not for power function, but for exponential function form of the PDF, such as the exponential distribution family [1] with PDF $e^{-x^{n}} n x^{-1+n}, x \in(0, \infty), n>0$, which is WeibullDistribution [n,1] or GammaDistribution $[1,1, n, 0]$ or MinStableDistribution $\left[1, \frac{1}{n},-\frac{1}{n}\right]$, will it behave similarly: the kurtosis divided by the squared skewness is the only choice which gives nonzero finite value when the skewness and kurtosis are infinite? Or will it have a critical value bigger than 2 ? We will see that it is the second case, and start the study from its central moment:

$$
\begin{aligned}
& \{C M[2], C M[3], C M[4]\}=\left\{- \text { Gamma }\left[1+\frac{1}{n}\right]^{2}+\text { Gamma }\left[1+\frac{2}{n}\right], 2 \text { Gamma }[1+\right. \\
& \left.\frac{1}{n}\right]^{3}-3 \text { Gamma }\left[1+\frac{1}{n}\right] \text { Gamma }\left[1+\frac{2}{n}\right]+\text { Gamma }\left[1+\frac{3}{n}\right],-3 \text { Gamma }\left[1+\frac{1}{n}\right]^{4}+ \\
& \text { 6Gamma }\left[1+\frac{1}{n}\right]^{2} \text { Gamma }\left[1+\frac{2}{n}\right]-4 \text { Gamma }\left[1+\frac{1}{n}\right] \text { Gamma }\left[1+\frac{3}{n}\right]+ \\
& \text { Gamma } \left.\left[1+\frac{4}{n}\right]\right\} .
\end{aligned}
$$

The goal of our asymptotic analysis is to find a simpler form for the limit. The sum of terms can be simplified by removing all terms that are of lower order of magnitude. 
Algebraic or more quickly numerical method can be used to find inequalities or to compare orders. For example, we can deduct either by $C M[2] \geq 0$ or from the numerical minimum NMinimize $\left[\left\{-\right.\right.$ Gamma $\left[\frac{1}{n}\right]^{2}+2 n$ Gamma $\left.\left.\left[\frac{2}{n}\right], n>0\right\},\{n, 1 / 10,1000\}\right]=$ $\{0.8425644753494974,\{n \rightarrow 1.6219726504389582\}\}$ that:

$2 n$ Gamma $\left[\frac{2}{n}\right]>$ Gamma $\left[\frac{1}{n}\right]^{2}$, when $n>0$.

This inequality is unique of the Gamma function, and is not hold for general log convex functions. It gives us idea or hint of the dominance of terms, then either by plot or by calculating symbolic limit of $\frac{\operatorname{Gamma}\left[\frac{1}{n}\right]^{2}}{2 n \mathrm{Gamma}\left[\frac{2}{n}\right]}$ we know the squared term in $C M[2]$ can be ignored.

We finally get the asymptotic expansion of (16) when $n \rightarrow 0$ from those simplifications and other simplifications such as using the formula $(a+n)^{\frac{a}{n}} \rightarrow e a^{\frac{a}{n}}$, where we cannot simply remove the $n$ in the sum without add the factor $e$ in:

$\operatorname{limit}_{n \rightarrow 0}\{C M[2], C M[3], C M[4]\} \sim\left\{\left(\frac{2}{e n}\right)^{\frac{2}{n}} \sqrt{4 \frac{\pi}{n}},\left(\frac{3}{e n}\right)^{\frac{3}{n}} \sqrt{6 \frac{\pi}{n}},\left(\frac{4}{e n}\right)^{\frac{4}{n}} \sqrt{8 \frac{\pi}{n}}\right\}$.

From (18) and from the $7^{\text {th }}$ order expansion of the Gamma $[x, y]$ at infinity followed by removing the minor terms we get:

$$
\begin{gathered}
\operatorname{limit}_{n \rightarrow 0}\left\{S, K, S F, \frac{K}{S^{\frac{4}{3}}}, S F 3[2]\right\} \sim \\
\left\{\left(\frac{6}{8}\right)^{\frac{1}{2}}\left(\frac{27}{8}\right)^{\frac{1}{n}}\left(\frac{n}{\pi}\right)^{\frac{1}{4}},\left(\frac{1}{2}\right)^{\frac{1}{2}}(16)^{\frac{1}{n}}\left(\frac{n}{\pi}\right)^{\frac{1}{2}}, \frac{2^{\frac{3}{2}}\left(\frac{1024}{729}\right)^{\frac{1}{n}}}{3}, \frac{2^{\frac{5}{6}\left(\frac{256}{81}\right)^{\frac{1}{n}} n^{1 / 6}}}{3^{\frac{2}{3}} \pi^{1 / 6}}, \frac{4^{\frac{1}{n}} \sqrt{n}}{4 \sqrt{\pi}}\right.
\end{gathered}
$$

There are generally wonders about the differences of the $S D$ and $M D$. The deviation of them as represented by $S F 3[2]$ is a measure of the convexity of the PDF, and since it involves absolute function, the calculations are more complex, so much so that its asymptotic expression cannot be validated by symbolic limit but only by plots or numerical evaluation for lists of values. The asymptotic approximation for $\frac{K}{S^{\frac{4}{3}}}$ is not as neat as $S F$ either.

Also from (18) we know that $\frac{K}{S^{a}} \sim 2^{-\frac{1}{2}+a} 3^{-\frac{a}{2}}\left(\frac{2^{4+3 a}}{3^{3 a}}\right)^{\frac{1}{n}} n^{\frac{1}{2}-\frac{a}{4}} \pi^{\frac{1}{4}(-2+a)}$. The solution of $\frac{2^{4+3 a}}{3^{3 a}}=1$ gives a critical point 2.279348388468605 that is larger than 2 : when a is above it, $\operatorname{limit}_{n \rightarrow 0} \frac{K}{S^{\mathrm{a}}} \sim 0$, but when a is equal to or below it, $\operatorname{limit}_{n \rightarrow 0} \frac{K}{S^{\mathrm{a}}} \sim \infty$. So this is an example we cannot see a nonzero finite limit number, and an example which has a critical value bigger than 2 .

From the minimum plot $\frac{K}{|S|^{\mathrm{a}}}$ in Fig. 4, we see that the asymptotic formula gives critical value very close to the original shape factors critical value 2.279882 . By numerical optimization, we find the minimum of $\frac{K}{|S|^{\mathrm{a}}}$ is 1.138705 when the minimum is bigger 
than 0 , complying with our guess in section 2.1. This numerical result is supported by the contour plot of the derivative of $\frac{K}{|S|^{\mathrm{a}}}$ with respective to distribution parameter n, Fig. 5 , where we see when a is fixed and between 2.279882 and $2.29975, \frac{K}{|S|^{\mathrm{a}}}$ has two minimum with the global minimum 0 attended when $n \rightarrow 0$.

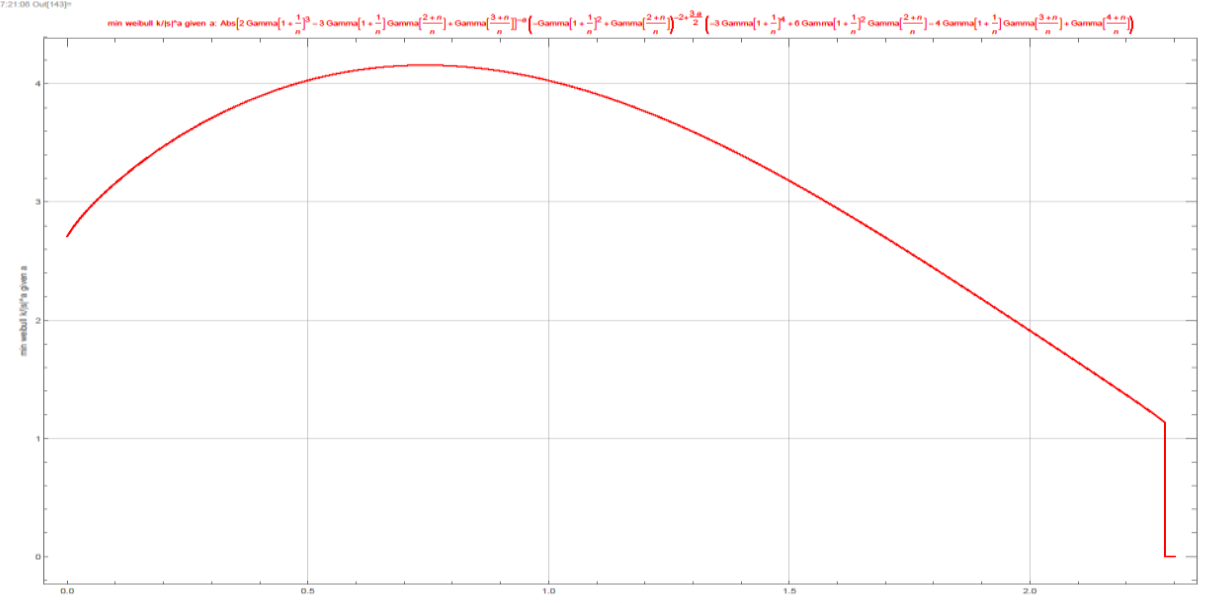

Fig. 4. Weibull distribution minimum $\frac{K}{|S|^{\mathrm{a}}}$ for given a. The horizontal axis is a.

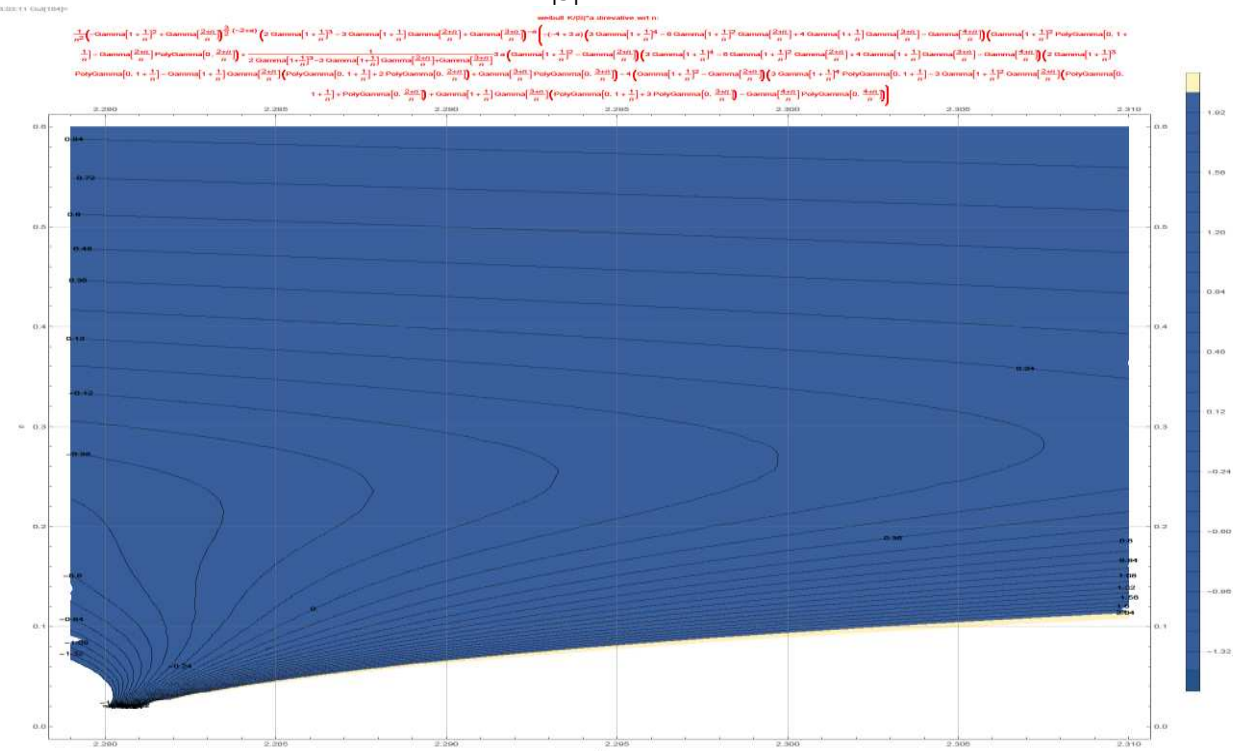

Fig. 5. Weibull distribution contour plot of $D\left[\frac{K}{|S|^{\mathrm{a}}}, n\right]$. The horizontal axis is a. When $\mathrm{a}=2$, the minimum of $\frac{K}{|S|^{\mathrm{a}}}$ is 1.9122718704899456695 , attended at $\mathrm{n}=$ 0.6411485565697524681 . 


\subsection{Kumaraswamy Distribution Part Two}

\section{Boundary $\boldsymbol{\beta}=\infty$}

Return to Kumaraswamy distribution, for a given $\alpha$, when $\beta \rightarrow \infty$, [1] formula (4)-(6) gives the value of the skewness, kurtosis, and the shape factor. We have a similar trick to (12) that:

if $\log (f(x)) x \rightarrow \log (\mathrm{a})$, then possibly $f(x) \sim \mathrm{a}^{\frac{1}{x}}$.

Use (20), and confirmed by both $4^{\text {th }}$ order and $1^{\text {st }}$ order asymptotic expansion of the Gamma function we get the asymptotic order of the boundary shape factor:

$\operatorname{limit}_{\alpha \rightarrow 0} \operatorname{limit}_{\beta \rightarrow \infty} S F \sim \frac{2^{\frac{3}{2}}\left(\frac{1024}{729}\right)^{\frac{1}{\alpha}}}{3}$.

It is a surprise that the shape factor formula (21) for Kumaraswamy distribution and formula (19) for Weibull distribution are the same while their PDF are very different.

From equation (13), at the boundary $\alpha=0$, the shape factor increase from 1 to $\infty$ when $\beta$ turns from 0 to $\infty$. From equation (21) and [1] equation (4) and (6), at the boundary $\beta=\infty$, the shape factor has a minimum value of 1.9122718704899369 when $\alpha=$ 0.6411485567602634 , increase to $\infty$ when $\alpha$ turns to 0 or 3.602349425719043 .

This minimum value for Kumaraswamy distribution is very unusual since it is at the same time the minimum shape factor of the MaxStableDistribution $[\mu, \sigma, \xi]$ and the GB2 distribution BetaPrimeDistribution[p,q, $\alpha, \beta]$ when $p=1$ ([1] Section 7.1 and Figure 26), three distributions with no relationship apparently.

One experience in this exploration is that when series expansion and heavy substitution are made, the final asymptotic form deducted or guessed need to be validated with the original expression, either by take the symbolic limit of the ratio, or by numerical evaluation of the ratio; different orders of the series expansion arriving at the same form is not enough to guarantee that the form is correct.

\section{Relationship with Weibull Distribution}

The identical asymptotic form and minimum value of the shape factor shows that the Kumaraswamy distribution when $\beta \rightarrow \infty$ and the Weibull distribution may be related. This guess is proved in Fig. 6 that they have identical skewness, kurtosis, and shape factor formulas. 


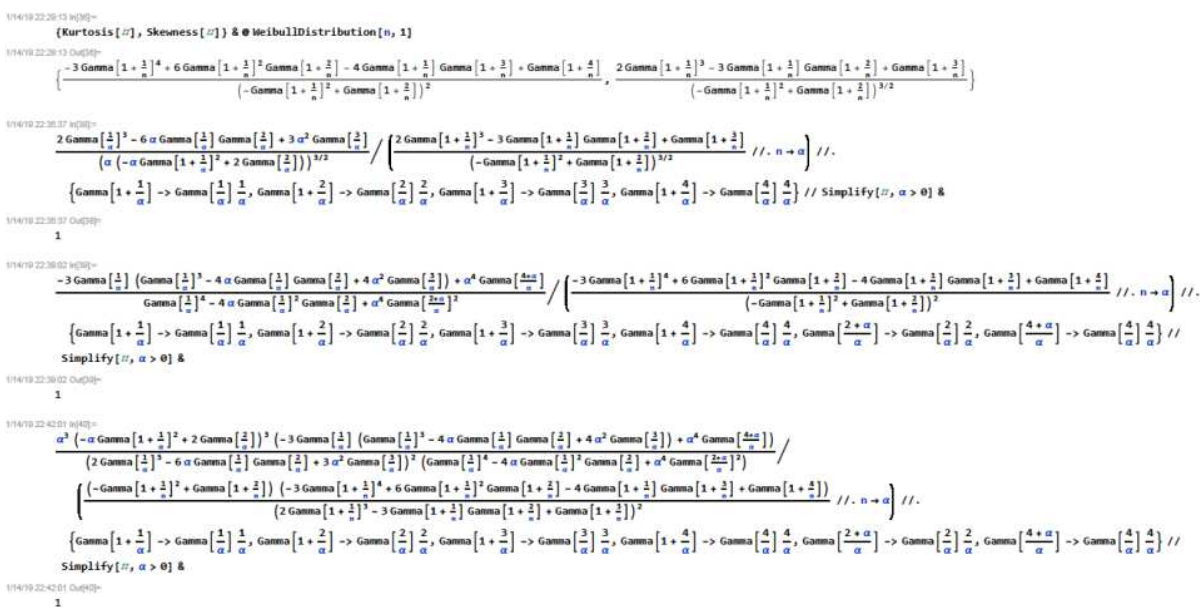

Fig. 6. Prove that Kumaraswamy $\beta \rightarrow \infty$ and Weibull distribution have identical S K SF.

Since Kumaraswamy distribution can be regarded heuristically as $\beta$ fold minimum distribution of the power distribution $x^{\alpha}$, when $\beta \rightarrow \infty$, we guess it should converge in distribution to some extreme value distribution [7][8], and Weibull distribution or the slightly general MinStableDistribution is just that extreme value distribution.

Diverse distributions converge to one of the three types of extreme value distribution, so boundary value analysis or asymptotic analysis of the shape factor should arrive at the same or a few typical simple form. We can call distributions with identical SF boundary value formulas asymptotically equivalent distributions, so that asymptotically equivalent distributions will have close or identical parameters when fit a given empirical distribution. This is non-trivial when their PDF/CDFs do not have clear relationships or similarities.

\section{Minimum Shape Factor Value for Given $\alpha$ or $\beta$}

For fixed $\beta$ and the Kumaraswamy distribution, unlike the Beta distribution, the lower boundary values are not the minimum values of the shape factor, which are attended at interior points, FindRoot are used to plot them, Fig.7. 


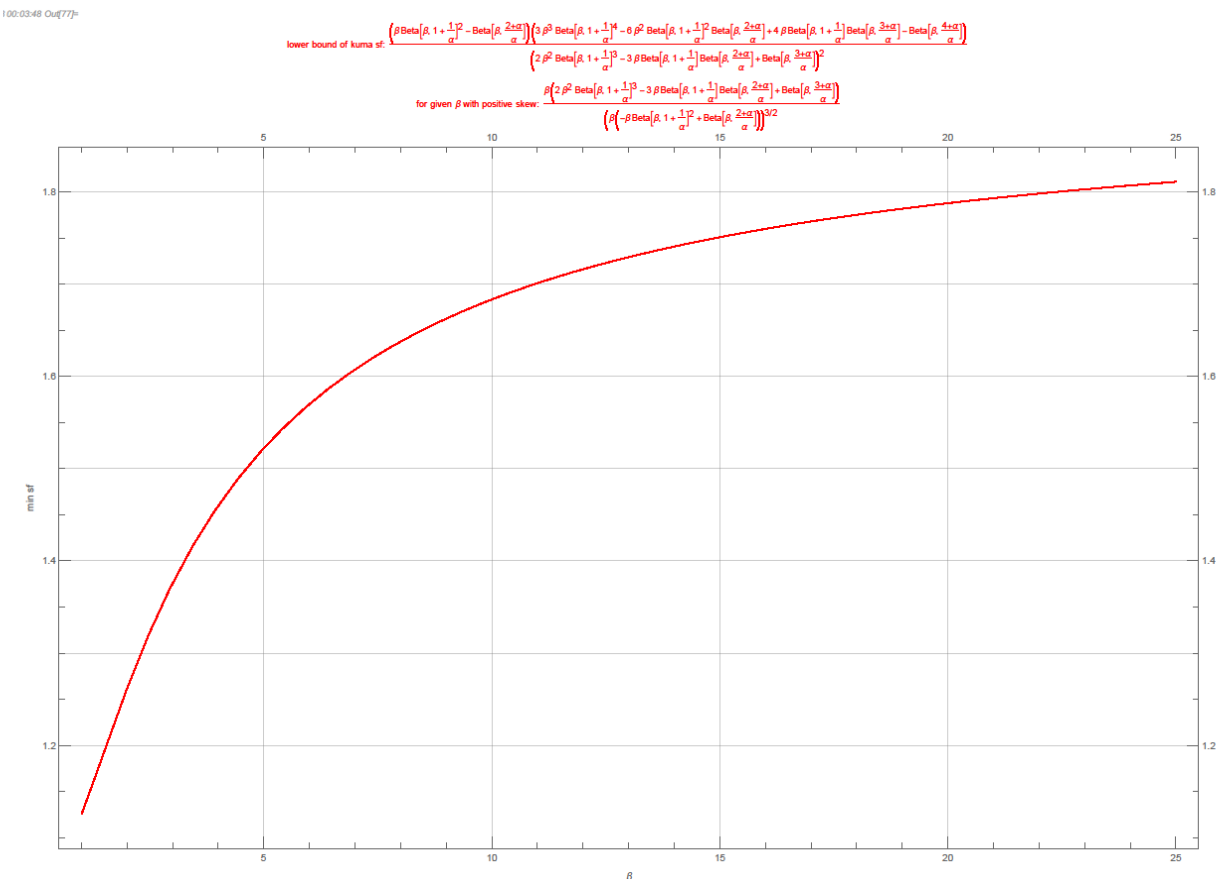

Fig. 7. Kumaraswamy distribution minimum shape factor for given $\beta$. The horizontal axis is $\beta$.

In Fig.7 we see that when $\beta$ increases from 0 to $\infty$, the minimum shape factor increases from 1 to 1.91227 , the minimum value of the shape factor at the boundary $\beta=\infty$.

For a given $\alpha$, the minimum shape factor is also taking place in interior point, and increases from 1 to $\infty$ as $\alpha$ increases from 0 to 3.602349, Fig. 8. Different directional minimum behaves differently. 


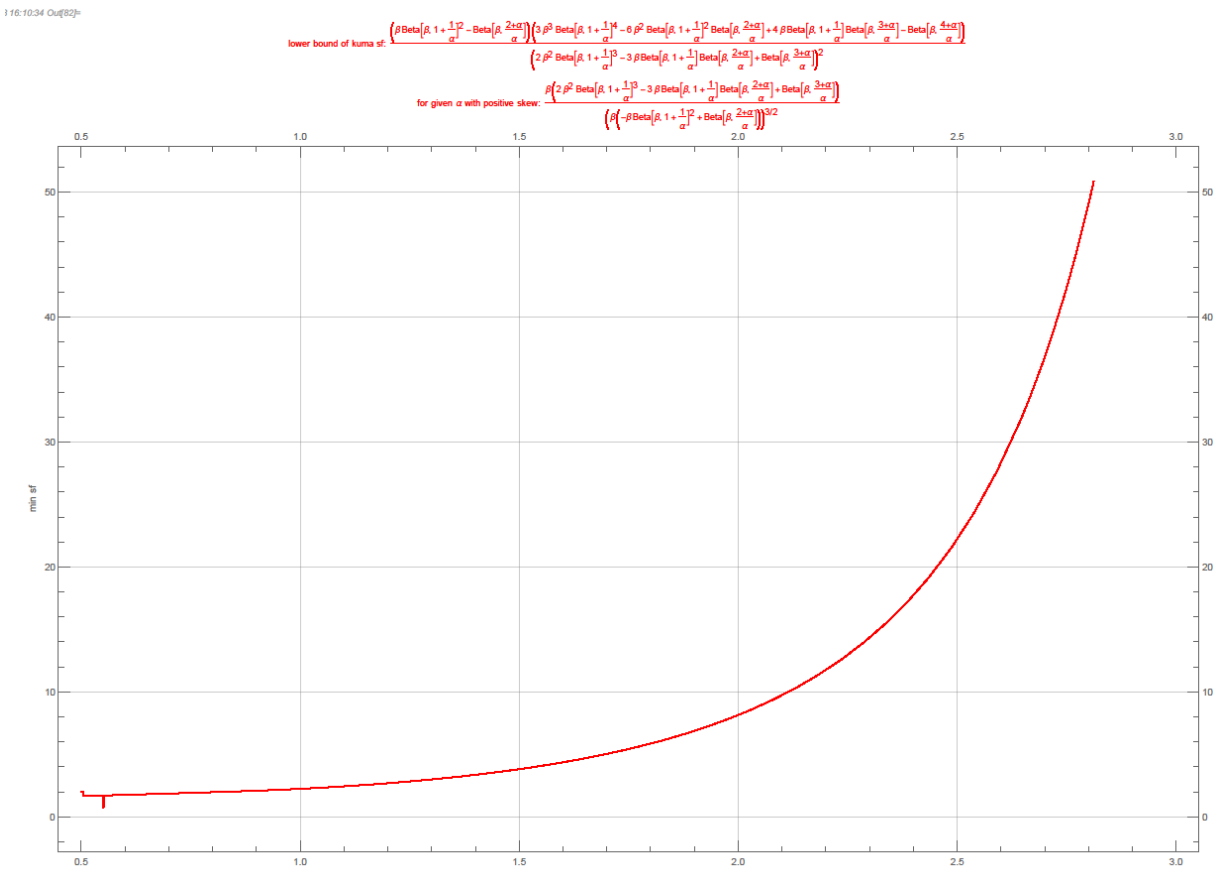

Fig. 8. Kumaraswamy distribution minimum shape factor for given $\alpha$. The horizontal axis is $\alpha$.

For given shape factor, Fig. 7 and Fig. 8 give the permissible parameters $\alpha$ and $\beta$ ranges.

\subsection{GB2 Distribution}

Asymptotic Expression When $\mathbf{q} \rightarrow \infty$

The GB2([5]), or BetaPrimeDistribution $[p, q, \alpha, \beta]$ shape factor turns to constant when $\mathrm{q} \rightarrow \infty$ from Figure 22 and 23 in [1]. From section 2.4 we see that the asymptotic expression or boundary value of the shape factors can be used as a hint for the original shape factors. So we will utilize asymptotic analysis of the shape factor not for its own sake but as an approximation or initial value to the original shape factor, followed by numerical correction or validation. Combined with affine transformation invariance, we can assume $q \rightarrow \infty, \beta=1$. From Gamma function $1^{\text {st }}$ order expansion at $\infty$ we guessed and proved by calculating symbolic limit that:

$\operatorname{limit}_{q \rightarrow \infty}\{C M[2], C M[3], C M[4]\}=$
$\left\{\operatorname{Gamma}[q]^{-2} \mathrm{e}^{-2 q} q^{-1+2 q-\frac{2}{\alpha}}, \operatorname{Gamma}[q]^{-3} \mathrm{e}^{-3 q} q^{-\frac{3}{2}+3 q-\frac{3}{\alpha}}, \operatorname{Gamma}[q]^{-4} \mathrm{e}^{-4 q} q^{-2+4 q-\frac{4}{\alpha}}\right\}$.

Divided by these factors that will cancel out by themselves we can get the asymptotic limit for $\mathrm{q} \rightarrow \infty$ : 


$$
\begin{aligned}
& \operatorname{limit}_{q \rightarrow \infty} S \sim \frac{2 \operatorname{Gamma}\left[p+\frac{1}{\alpha}\right]^{3}-3 \operatorname{Gamma}[p] \operatorname{Gamma}\left[p+\frac{1}{\alpha}\right] \operatorname{Gamma}\left[p+\frac{2}{\alpha}\right]+\operatorname{Gamma}[p]^{2} \operatorname{Gamma}\left[p+\frac{3}{\alpha}\right]}{\left(-\operatorname{Gamma}\left[p+\frac{1}{\alpha}\right]^{2}+\operatorname{Gamma}[p] \operatorname{Gamma}\left[p+\frac{2}{\alpha}\right]\right)^{\frac{3}{2}}}, \\
& \operatorname{limit}_{q \rightarrow \infty} K \sim-\frac{1}{\left(-\operatorname{Gamma}\left[p+\frac{1}{\alpha}\right]^{2}+\operatorname{Gamma}[p] \operatorname{Gamma}\left[p+\frac{2}{\alpha}\right]\right)^{2}}\left(3 \operatorname{Gamma}\left[p+\frac{1}{\alpha}\right]^{4}-6 \operatorname{Gamma}[p] \operatorname{Gamma}[p+\right. \\
& \left.\left.\frac{1}{\alpha}\right]^{2} \operatorname{Gamma}\left[p+\frac{2}{\alpha}\right]+4 \operatorname{Gamma}[p]^{2} \operatorname{Gamma}\left[p+\frac{1}{\alpha}\right] \operatorname{Gamma}\left[p+\frac{3}{\alpha}\right]-\operatorname{Gamma}[p]^{3} \operatorname{Gamma}\left[p+\frac{4}{\alpha}\right]\right), \\
& \operatorname{limit}_{q \rightarrow \infty} S F \sim \frac{\operatorname{Gamma}\left[p+\frac{1}{\alpha}\right]^{2}-\operatorname{Gamma}[p] \operatorname{Gamma}\left[p+\frac{2}{\alpha}\right]}{\left(2 \operatorname{Gamma}\left[p+\frac{1}{\alpha}\right]^{3}-3 \operatorname{Gamma}[p] \operatorname{Gamma}\left[p+\frac{1}{\alpha}\right] \operatorname{Gamma}\left[p+\frac{2}{\alpha}\right]+\operatorname{Gamma}[p]^{2} \operatorname{Gamma}\left[p+\frac{3}{\alpha}\right]\right)^{2}}\left(3 \operatorname{Gamma}\left[p+\frac{1}{\alpha}\right]^{4}-\right. \\
& 6 \operatorname{Gamma}[p] \operatorname{Gamma}\left[p+\frac{1}{\alpha}\right]^{2} \operatorname{Gamma}\left[p+\frac{2}{\alpha}\right]+4 \operatorname{Gamma}[p]^{2} \operatorname{Gamma}\left[p+\frac{1}{\alpha}\right] \operatorname{Gamma}\left[p+\frac{3}{\alpha}\right]- \\
& \left.\operatorname{Gamma}[p]^{3} \operatorname{Gamma}\left[p+\frac{4}{\alpha}\right]\right) .
\end{aligned}
$$

The simpler formula in the right side of (23)-(25) for S, K, and SF which only involve parameters $\mathrm{p}$ and $\alpha$ will be our new starting point for studying the minimum and boundary tendencies, and we will call them SB, KB, and SFB, the boundary values of $\mathrm{S}, \mathrm{K}$, and $\mathrm{SF}$ for $\mathrm{q}=\infty$.

First we have some symbolic limit values for them:

$$
\begin{aligned}
& \operatorname{limit}_{p \rightarrow 0} S F B=\frac{\operatorname{Gamma}\left[\frac{2}{\alpha}\right] \mathrm{Gamma}\left[\frac{4}{\alpha}\right]}{\operatorname{Gamma}\left[\frac{3}{\alpha}\right]^{2}}, \\
& \operatorname{limit}_{\alpha \rightarrow \infty} S F B=\frac{\text { PolyGamma }[1, p]\left(3 \text { PolyGamma }[1, p]^{2}+\text { PolyGamma }[3, p]\right)}{\text { PolyGamma }[2, p]^{2}}, \\
& \operatorname{limit}_{p \rightarrow \infty} S F B=\text { ComplexInfinity, } \operatorname{limit}_{\alpha \rightarrow 0} S F B=\infty .
\end{aligned}
$$

When $\mathrm{p}$ increases from 0 to $\infty$, equation (27) increases from 2.25 almost linearly to $\infty$. When $\alpha$ increases from 0 to $\infty$, equation (26) decreases from $\infty$ to 1.125 . The two directional limits of SFB at the corner of $p=0$ and $\alpha=\infty$ are different.

\section{Minimum Shape Factor Given p}

Now we reduced the parameters numbers to 2, we can similarly use contour plot, partial derivative contour plot, and partial derivative zero points to get minimum shape factor values. For fixed $\mathrm{p}$ and $\alpha$, when $\mathrm{q} \rightarrow \infty$, from the contour plot we see the SF is decreasing, a justification for using $\mathrm{q} \rightarrow \infty$ asymptotic values to calculate the minimum shape factor.

$\mathrm{S}$ and SF 3D plot with mesh can show us overall values distribution of SF, Fig. 8, Fig. 9; from them we can see that the positive SF region is at the bottom left portion where $\alpha$ is small, and for given $p$ the minimum SF is attended in interior point of $\alpha$. 


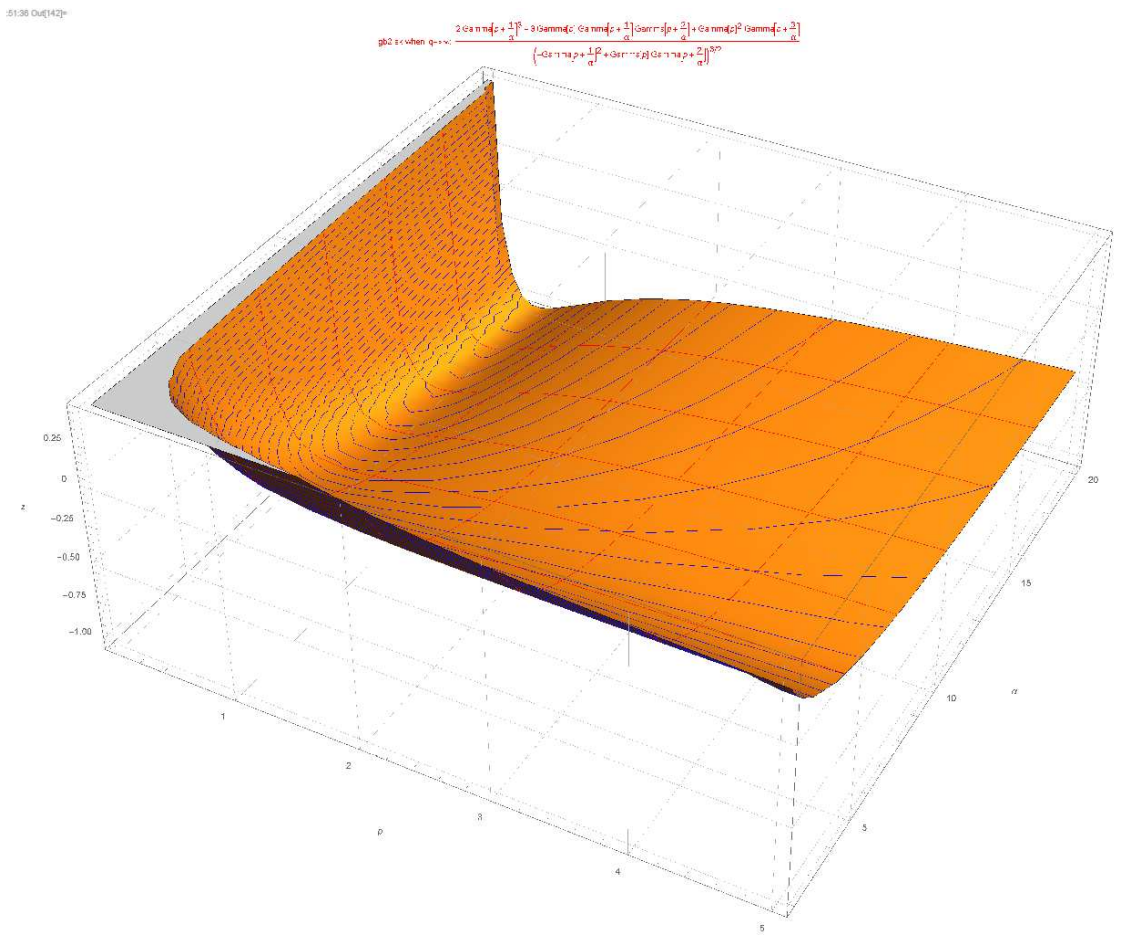

Fig. 9. GB2 Skewness 3D contour plot at $\mathrm{q}=\infty$. 


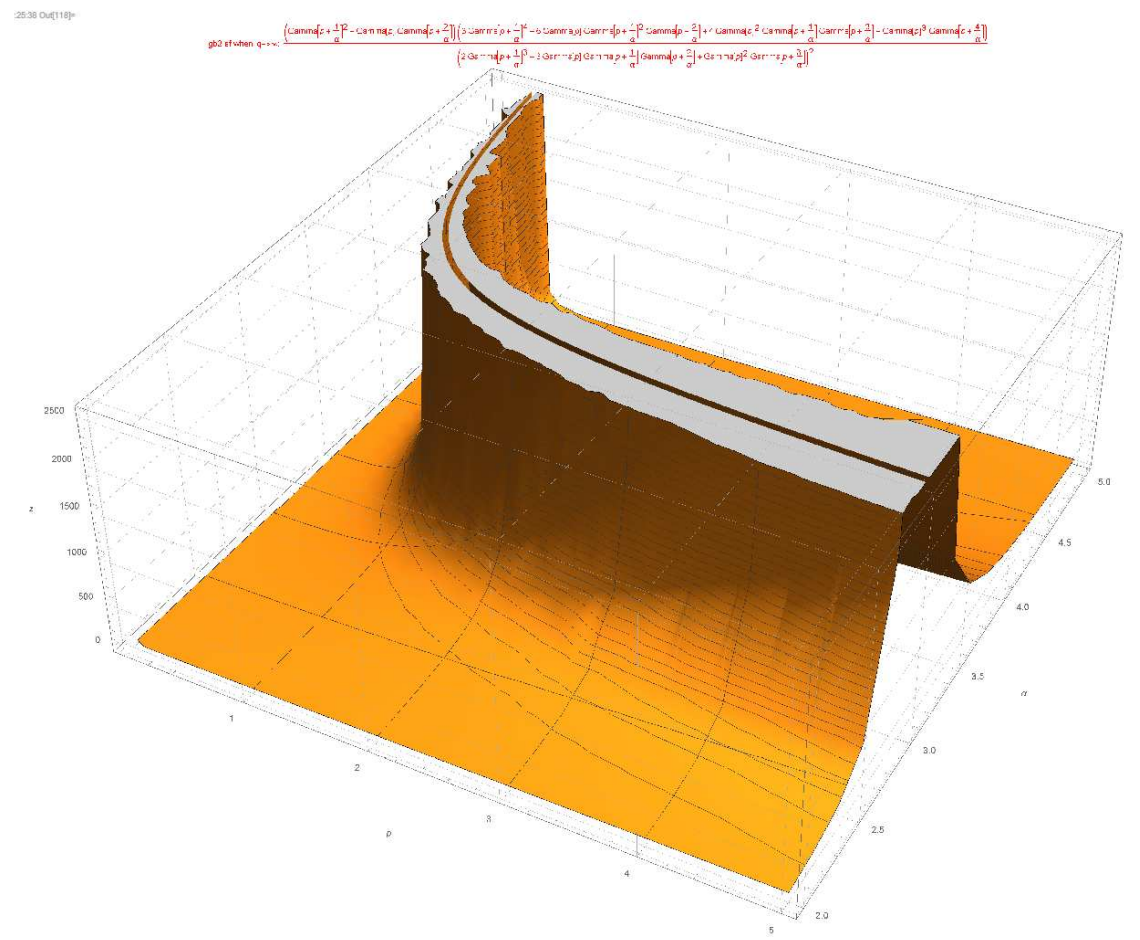

Fig. 10. GB2 shape factor $3 D$ contour plot at $q=\infty$.

Take the SF asymptotic formula (25) or SFB partial derivative with respect to $\alpha$, solve to get its zero points and the corresponding SFB value, plot it against the empirical minimum SF formula (7) from [1], in Fig. 11. That these two curves are very close can be regarded as both validations for our asymptotic formula (25) and the empirical formula (7) from [1]. 


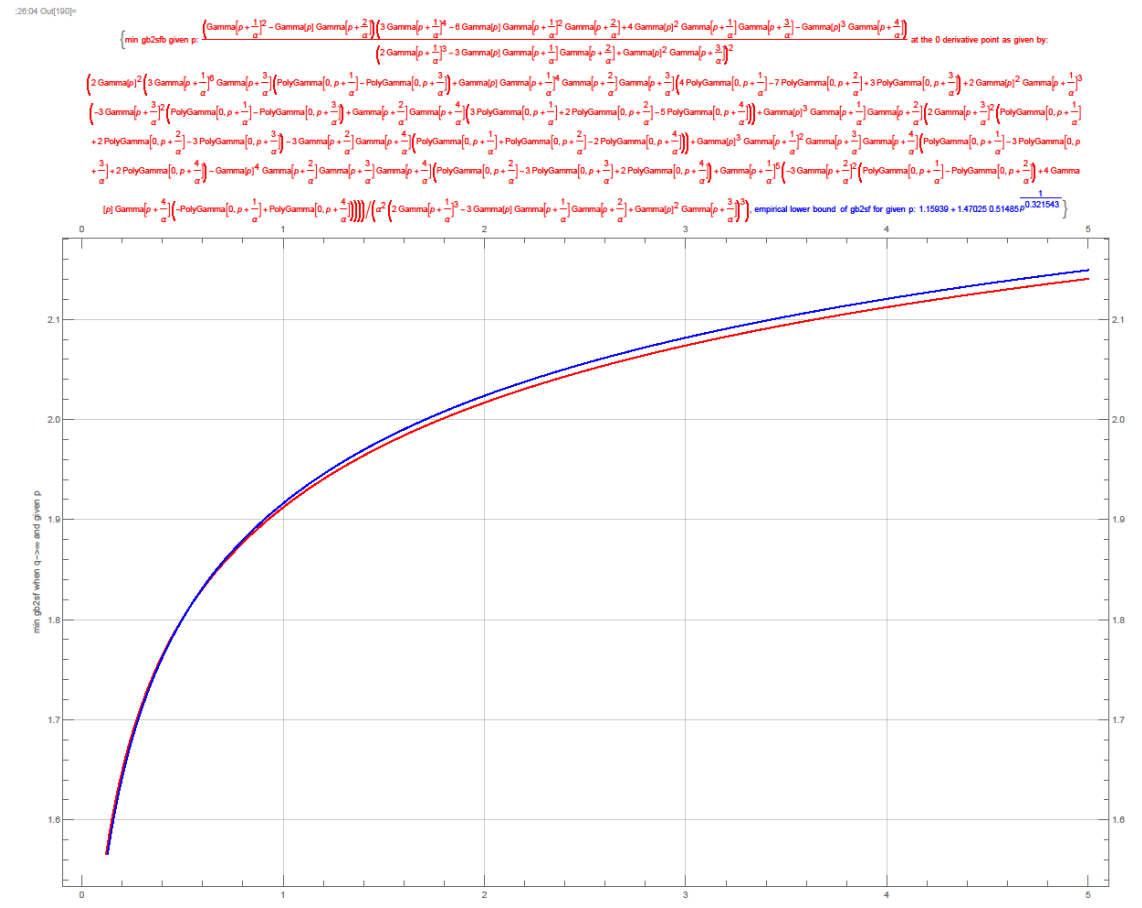

Fig. 11. GB2 minimum SF plot for given $p$, either from asymptotic expression when $q \rightarrow \infty$ or from empirical formula for numerical optimization results.

The two curves in Fig. 11 have two intersection at $p \approx 0.0016$ and $p \approx 0.52$, inside the interval $[0.0016,0.52]$ the empirical curve is slightly lower, and outside of it the asymptotic formula is lower. Checking against the original numerical optimization results shows that when $\mathrm{p}<1$ the asymptotic formula gives better match than the empirical formula, in this case the "human learning" is better than the machine learning from Mathematica FindFormula.

This minimum shape factor through asymptotic expression converges to 1.125 when $\mathrm{p} \rightarrow 0$, and to 2.4881 when $\mathrm{p} \rightarrow \infty$.

\section{Minimum Shape Factor Given $\alpha$}

We can get the minimum shape factor for given $\alpha$ similarly by using SFB partial derivative with respect to $p$. But there is a max $\alpha=0.6379212899193917$, below it, the minimum take place at the zero derivative point, above it, all the partial derivative is positive and the minimum shape factor is attended at the boundary of $\mathrm{p}=0$ through formula (26). So the plot of the minimum shape factor given $\alpha$ has two portion which intersect at the mentioned $\alpha$ value with corresponding shape factor value 1.8147359390703104, Fig. 12. 


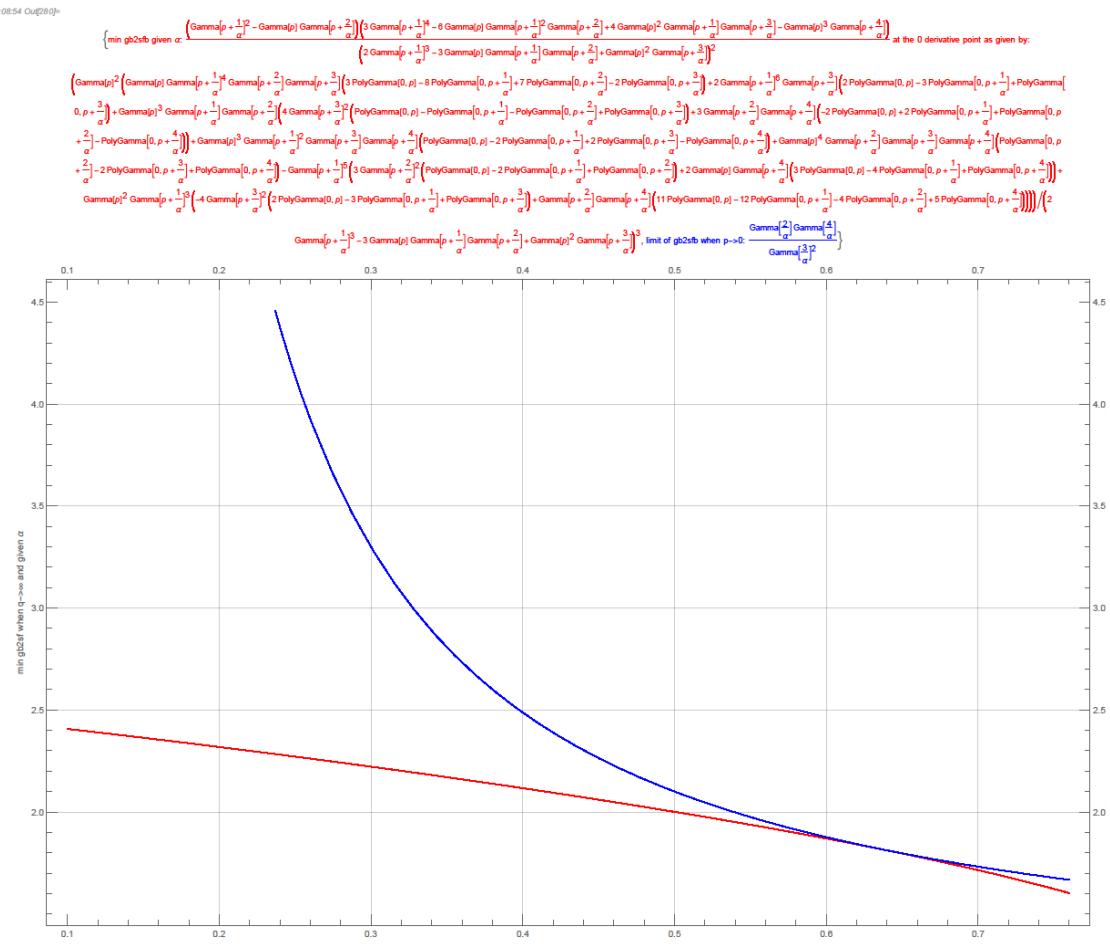

Fig. 12. GB2 minimum SF plot for given $\alpha$, from SFB zero derivative point value or boundary value when $\mathrm{p} \rightarrow 0$.

The composite plot in Fig.12 is checked against the empirical plot Fig. 27 in [1] for the range of $\alpha$ from 0.5 to 1 : they matched very well. The tendency of the minimum shape factor given $\alpha$ as shown in Fig. 12 is decreasing from $\infty$ to 1.125 when $\alpha$ turns from 0 to $\infty$.

Fig. 11 and Fig.12 can be used to validate the parameter or find the parameters $p$ and $\alpha$ range when the shape factor is given.

One lesson learned in using series expansion or asymptotic expansion to study the limit of shape factor in the GB2 case is that different order expansions may give different results. For example, in studying the SFB limit when $\mathrm{p} \rightarrow \infty$, if we use the $0^{\text {th }}$ order expansion of the Gamma function at $\infty$, we get $\operatorname{limit}_{p \rightarrow \infty} S F B \sim-\frac{1}{25} p \alpha$, an absurd negative number; if we use $1^{\text {st }}$ order expansion, and the substitution $\left(p+\frac{z}{\alpha}\right)^{-o+n\left(p+\frac{z}{\alpha}\right)} \rightarrow$ $e^{\frac{n z}{\alpha}} p^{-o+n\left(p+\frac{z}{\alpha}\right)}$, we get $\operatorname{limit}_{p \rightarrow \infty} S F B \sim-\frac{1}{4 \alpha} p^{-2}$, different but still negative. But for the $2^{\text {nd }}$, $3^{\text {rd }}, 4^{\text {th }}$, and $5^{\text {th }}$ order expansions, we get the same $\operatorname{limit}_{p \rightarrow \infty} S F B \sim \frac{4}{3}$. We may hurriedly conclude that the expansion converged when using above $2^{\text {nd }}$ order expansions. Symbolic calculation is useful, but is also error prone, such as when substitutes are made of terms 
in a summation expression. So it need to be confirmed by other means, such as numerical calculation and graphical plot. Plot of the $5^{\text {th }}$ order expansion SFB crashed Mathematica kernel, and numerical calculation caused overflow. It is found that Mathematica cannot calculate Gamma[10.^14] in arbitrary-precision arithmetic due to a restriction of maximum numbers allowed in this format. That may be why its plots have many void portions. So verification by alternative software is desired: there is a package MPMATH in SYMPY that can be tested in IPython, which can calculate $\operatorname{gamma}\left(10^{* * 14}\right)$ or even gamma(10**100). For $\alpha=1$, p increasing, MPMATH calculated SFB is also increasing and follows some pattern until $\mathrm{p}=10 * * 21$, after that the calculated SFB fluctuates between positive and negative numbers; for $\mathrm{p}=10 * * 55$, $10 * * 100000,10 * * 1000000$, it gives 0 : results hard to reconcile.

When plot the zero value contour in the parameter space of $\mathrm{p}$ and $\alpha$ of the partial derivatives of SFB with respective to $\alpha$ and $p$, we see that the former is higher than the latter, Fig.13.
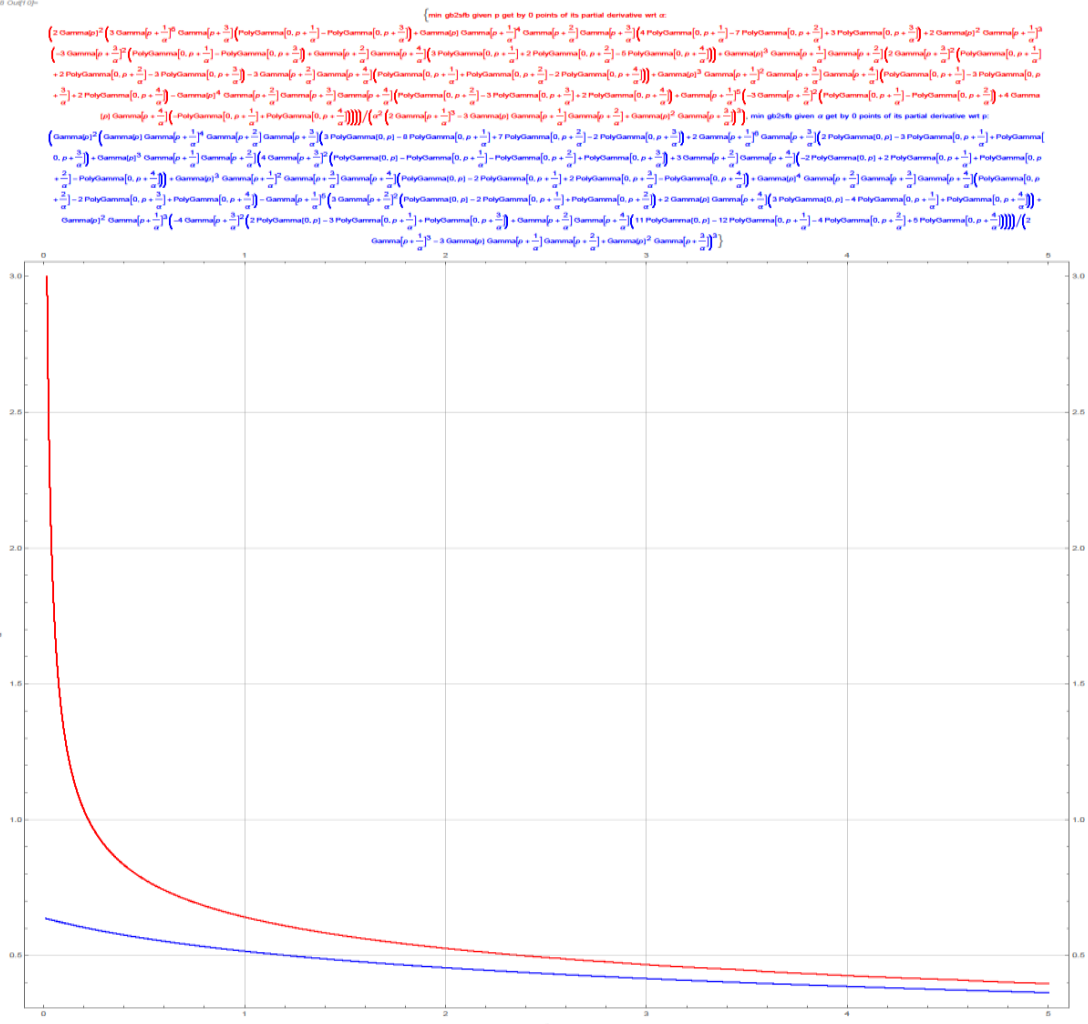

Fig. 13. $D[S F B, \alpha]$ and $D[S F B, p] 0$ contour plot.

Combine Fig. 13 and Fig.11 we know for given $\alpha$ sufficiently small, the SFB will be larger than 2.4881. So we may incline to think that $\infty$ rather than $\frac{4}{3}$ is the limit when $\mathrm{p} \rightarrow \infty$. We will leave that as a mystery to be solved. 


\section{Characteristic Number}

From equation (22) take more symbolic limit we get:

$$
\begin{aligned}
& \operatorname{limit}_{q \rightarrow \infty} C M[2] \sim q^{-\frac{2}{\alpha}} \frac{-\operatorname{Gamma}\left[p+\frac{1}{\alpha}\right]^{2}+\operatorname{Gamma}[p] \operatorname{Gamma}\left[p+\frac{2}{\alpha}\right]}{\operatorname{Gamma}[p]^{2}}, \\
& \operatorname{limit}_{q \rightarrow \infty} C M[3] \sim q^{-\frac{3}{\alpha}} \frac{2 \mathrm{Gamma}\left[p+\frac{1}{\alpha}\right]^{3}-3 \operatorname{Gamma}[p] \operatorname{Gamma}\left[p+\frac{1}{\alpha}\right] \operatorname{Gamma}\left[p+\frac{2}{\alpha}\right]+\operatorname{Gamma}[p]^{2} \operatorname{Gamma}\left[p+\frac{3}{\alpha}\right]}{\operatorname{Gamma}[p]^{3}} \\
& \operatorname{limit}_{q \rightarrow \infty} C M[4] \sim \frac{q^{-\frac{4}{\alpha}}}{\operatorname{Gamma}[p]^{4}}\left(-3 \operatorname{Gamma}\left[p+\frac{1}{\alpha}\right]^{4}+6 \operatorname{Gamma}[p] \operatorname{Gamma}[p+\right. \\
& \left.\frac{1}{\alpha}\right]^{2} \operatorname{Gamma}\left[p+\frac{2}{\alpha}\right]-4 \operatorname{Gamma}[p]^{2} \operatorname{Gamma}\left[p+\frac{1}{\alpha}\right] \operatorname{Gamma}\left[p+\frac{3}{\alpha}\right]+ \\
& \left.\operatorname{Gamma}[p]^{3} \operatorname{Gamma}\left[p+\frac{4}{\alpha}\right]\right) .
\end{aligned}
$$

From equation (29)-(31) and similarly by working with limit of each individual factors for a production expression we get:

$$
\begin{aligned}
& \operatorname{limit}_{p \rightarrow 0} \operatorname{limit}_{q \rightarrow \infty} \frac{K}{S^{\mathrm{a}}} \sim \operatorname{Gamma}[p]^{1-\frac{\mathrm{a}}{2}} \operatorname{Gamma}\left[\frac{2}{\alpha}\right]^{-2+\frac{3 \mathrm{a}}{2}} \operatorname{Gamma}\left[\frac{3}{\alpha}\right]^{-\mathrm{a}} \operatorname{Gamma}\left[\frac{4}{\alpha}\right], \\
& \operatorname{limit}_{\alpha \rightarrow 0} \operatorname{limit}_{q \rightarrow \infty} \frac{K}{S^{\mathrm{a}}} \sim 2^{\frac{3 \mathrm{a} p-\mathrm{a}-1}{2}} 3^{\mathrm{a}\left(\frac{1}{2}-p\right)} \pi^{\frac{\mathrm{a}-2}{4}}\left(\frac{2^{4+3 \mathrm{a}}}{3^{3 \mathrm{a}}}\right)^{\frac{1}{\alpha}} \alpha^{\frac{(\mathrm{a}-2)(2 p-1)}{4}} \operatorname{Gamma}[p]^{1-\frac{\mathrm{a}}{2}} .
\end{aligned}
$$

From equation (32) we know the characteristic number of GB2 distribution is still 2: whose $\min \frac{K}{s^{\mathrm{a}}} \rightarrow 0$ when $\mathrm{a}>2, \mathrm{q} \rightarrow \infty$, and $\mathrm{p} \rightarrow 0$.

Equation (33) says that at the boundary of $\alpha=0$ and $q=\infty$, an identical to the omnipresent Weibull distribution critical value $a=2.279348388468605$ exit: above it, $\operatorname{limit}_{\alpha \rightarrow 0} \operatorname{limit}_{q \rightarrow \infty} \frac{K}{S^{\mathrm{a}}} \sim 0$, but below it, $\operatorname{limit}_{\alpha \rightarrow 0} \operatorname{limit}_{q \rightarrow \infty} \frac{K}{S^{\mathrm{a}}} \sim \infty$. So the $\alpha=0$ and the $\mathrm{p}=0$ boundaries have different directional critical values with the $\mathrm{p}=0$ boundary one smaller and gives the global characteristic number 2 for GB2.

\section{Conclusion and Discussions}

The conditional minimum of the shape factor for given parameter value or given expression value such as the skewness is useful, but its plot can usually only be obtained through numerical method (as in [1][2]). The simplification of the shape factor through asymptotic approximation can provide a deterministic way of solving the conditional minimum problem. The numerical and analytical method are thus checking and validating each other. In the process of those boundary or limit and minimum analysis, some characteristics of the shape factor (the characteristic number), as well as mysterious relationships of distributions, such as those between Kumaraswamy and Weibull distributions, and between GB2 and Weibull distributions, BetaPrimeDistributio $[1, \infty, \alpha, 1] \approx$ WeibullDistribution $[\alpha, 1] \approx$ KumaraswamyDistribution $[\alpha, \infty]$, in the sense of identical S, K, and SF formulas, are discovered. 
GB1 distribution, similar to GB2 distribution, has simpler form of moment than central moment; those kind of shape factor by moment, such as $\frac{M[2] * M[4]}{M[3]^{2}}$, is easier to work at, and arrive at identical boundary or asymptotic limit formulas as we get of GB2 or Kumaraswamy distribution. The asymptotic limit seems even out the differences between moment and central moment in this case.

So whenever asymptotic limit can be calculated and has simpler form, it will be an invaluable tool for studying the original shape factor. This substitute method is also applicable when the limit of distribution PDF/CDF is hard to get, we can work on the SF limit instead; or when some but not all of S, K, and SF have infinite limit, we can change/modify to study the one with finite limit which can reveal additional information of the distribution ("structure inside the singularity").

Heuristically or by analogy we can think $\mathrm{S}$ as a first order derivative, $\mathrm{K}$ as a shifted first order derivative, and SF as a second order derivative, describing the convexity or curvature of the distribution PDF, so in some cases SF should have simpler form than $\mathrm{S}$ or $\mathrm{K}$, a reason for using it as the alternative.

The method in Fig. 2 can be used to study GB2 minimum shape factor with given product of $p \alpha$, and we guess the peak in [1] Fig. 27 is the impact of the zero value contour curve of the skewness. Some deduction of the asymptotic value of the shape factor of $\mathrm{GH}$ is in [2], but the detailed study for all these will be in a subsequent paper.

\section{Conflict of Interest}

The author declare no conflict of interest.

\section{References}

1. Wang FX (2018) What determine EP curve shape? doi:10.13140/RG.2.2.30056.11523

2. Wang FX (2019) What determines EP curve shape? In: Dr. Bruno Carpentieri (ed) Applied Mathematics. https://cdn.intechopen.com/pdfs/64962.pdf

3. Hardy GH, Wright EM (1979) An Introduction to the Theory of Numbers, 5th ed. Oxford, England: Clarendon Press

4. Marichev O, Trott M (2013) The Ultimate Univariate Probability Distribution Explorer. http://blog.wolfram.com/2013/02/01/the-ultimate-univariate-probability-distribution-explorer/. Accessed 6 June 2018

5. McDonald JB, Sorensen J, Turley PA (2011) Skewness and kurtosis properties of income distribution models. LIS Working Paper Series, No. 569. Review of Income and Wealth. doi:10.1111/j.1475-4991.2011.00478.x.

https://pdfs.semanticscholar.org/eabd/0599193022dfc65ca00f28c8a071e43edc32.pdf

6. McDonald JB (1984) Some Generalized Functions for the Size Distribution of Income. Econometrica 52(3):647-663

7. Embrechts P, Kluppelberg C, Mikosch T (1997) Modelling Extremal Events for Insurance and Finance. Springer. doi:10.1007/978-3-642-33483-2

8. McNeil AJ, Frey R, Embrechts P (2015) Quantitative Risk Management: Concepts, Techniques and Tools. Princeton University Press, Princeton, NJ, USA. 